\title{
Fluorescent detection of coenzyme A by analyte-induced aggregation of a cationic conjugated polymer
}

\author{
CHENG MingChao ${ }^{1}$, QIANG XiHuai ${ }^{*} \&$ DU ChiMin²* \\ ${ }^{1}$ College of Resource \& Environment, Shaanxi University of Science and Technology, Xi'an 710021, China; \\ ${ }^{2}$ Changzhou Sijie Biochemical Ltd, Changzhou 213022, China
}

Received July 2, 2012; accepted August 24, 2012; published online March 11, 2013

\begin{abstract}
A new cationic conjugated polymer was designed and synthesized to optically discriminate coenzyme $\mathrm{A}(\mathrm{CoA})$ among structurally similar biomolecules, ATP, ADP and AMP. The analyte-induced aggregation of the conjugated polymer by $\pi$-stacking between their main chains leads to the fluorescence quenching. Except for the similar adenosine and phosphate moieties as those in ATP, ADP and AMP, the CoA molecule also includes a long side chain that is favorable for hydrophobic interactions. Thus, CoA can form a complex with oppositely charged conjugated polymer by cooperative electrostatic and hydrophobic interactions, whereas ATP, ADP and AMP form the complexes with oppositely charged conjugated polymer mainly by electrostatic interactions. The increase of the ion strength of the assay solution screens the electrostatic attractions, and the remaining hydrophobic interactions dominate the formation of PFP-PTF/CoA complex. At this case, the quenching efficiency of PFP-PTF by CoA is much higher than that by ATP, ADP and AMP, which impart the PFP-PTF to sense CoA from these interferencing species.
\end{abstract}

conjugated polymers, coenzyme A, biosensor, complex, aggregates, fluorescence quenching

Citation: Cheng M C, Qiang X H, Du C M. Fluorescent detection of coenzyme A by analyte-induced aggregation of a cationic conjugated polymer. Chin Sci Bull, 2013, 58: 1256-1261, doi: 10.1007/s11434-013-5740-3

Since the coenzyme A (CoA) was discoved fifty years ago [1], it has been found to be concerned with many kinds of biochemical reactions [2]. One of the most important functions of $\mathrm{CoA}$ is the transfering of acyl groups in biological systems. It also serves multiple functions in a wide variety of metabolisms, such as glycolysis, fatty acid $\beta$-oxidation, and biosynthetic pathways utilizing acetyl-CoA [3]. It has been proved that among the known enzymes, about $4 \%$ of them require $\mathrm{CoA}$ or $\mathrm{CoA}$ ester as substrates [4,5]. CoA exists in plants, mammalians and microbial species [6-8]. The lack of CoA in the human body can cause deterioration of the immune system, anxiety, fatigue, impaired sense of balance, depression, irritability, fatigue and aging $[9,10]$. Nowadays CoA has been applied broadly in clinic to treat atherosclerosis, fatty liver, and so on [11]. On the other hand, very few chemical or biological sensors have been developed to sense CoA. The existing methods to detect CoA are by a reversed-phase high-performance liquid chromatography (HPLC) after the separation and centrifugation from the tissue [12-14]. After purification the sample solutions were injected into the HPLC to detect. The lowest detection limit was 3 pmol [13].

Recently, water-soluble conjugated polymers have got lots of attention as novel optical probes in sensitive biochemical sensors in view of their signal amplification function [15-19]. They have been used to detect nucleic acids, proteins, inorganic ions and biologically small active molecules [20-33]. In this paper, we prepare a new cationic conjugated polymer, that is utilized to optically discriminate CoA among structurally similar biomolecules based on analyte-induced aggregation of the conjugated polymer.

*Corresponding author (email: qiangxihuai@163.com) 


\section{Experimental}

\subsection{Materials and instrumentations}

All reagents were purchased from Aldrich or Alfa-Aesar Chemical Co. and used without further purification. Monomers $\mathbf{1}, \mathbf{2}$ and $\mathbf{3}$ were prepared according to the published literature procedures [34]. ${ }^{1} \mathrm{H}$ NMR spectra were obtained with a Bruker AV400 instrument. UV-vis absorption spectra were taken on a JASCO V-550 spectrophotometer. The gel permeation chromatography (GPC) measurements were performed on Water-410 system against polystyrene standard with THF as eluent. Linear light-scattering spectra were obtained from Hitachi F-4500 spectrofluorometer. Fluorescence measurements were obtained in $3 \mathrm{~mL}$ quartz cuvettes at room temperature using Hitachi F-4500 spectrofluorometer equipped with a Xenon lamp excitation source. The excitation wavelength is $361 \mathrm{~nm}$. The water was purified using a Millipore filtration system.

\subsection{Synthesis}

(1) Synthesis of precursor copolymer (4). The mixture of compound 1 (33.6 mg, $0.1 \mathrm{mmol}$ ), compound 2 (30.3 mg, $0.1 \mathrm{mmol}$ ), compound 3 (148.8 $\mathrm{mg}, 0.2 \mathrm{mmol}$ ) and sodium carbonate $(2 \mathrm{~mol} / \mathrm{L})$ in $15 \mathrm{~mL}$ of THF was degassed, and then $\mathrm{PdCl}_{2}$ (dppf) (dppf $=1,1$ '-bis(diphenylphosphine)ferrocene) was added to the mixture under $\mathrm{N}_{2}$. And the resulting mixture was stirred at $85^{\circ} \mathrm{C}$ for $36 \mathrm{~h}$ under $\mathrm{N}_{2}$ atmosphere. After cooling to room temperature (RT), $100 \mathrm{~mL}$ of distilled water was added to quench the reaction and the mixture was extracted with chloroform. After the organic solvent was evaporated, the residue was precipitated into $\mathrm{MeOH}$. The crude polymer was precipitated from $\mathrm{CHCl}_{3}$ into $\mathrm{MeOH}$ twice and dried under vacuum to give the product as a yellow solid. ${ }^{1} \mathrm{H}$ NMR (DMSO- $d_{6}, 400 \mathrm{MHz}$ ): $\delta_{\mathrm{ppm}}=7.15-7.83$ (br), 3.35-3.48 (br), 1.89-2.05 (br), 1.411.48 (br), 1.12-1.31 (br), 0.72-0.91 (br). GPC: $M_{\mathrm{n}}=15550$, $M_{\mathrm{w}}=70290, \mathrm{PDI}=4.52$.

(2) Synthesis of copolymer PFP-PTF. The aqueous trimethylamine $(33 \%)$ was added to the solution of neutral copolymer 4 in THF by dropwise at RT. After stirring for overnight the solvent was evaporated. The residue solid was precipitated from acetone to give a brown powder. ${ }^{1} \mathrm{H} \mathrm{NMR}$ (DMSO-d 6 , $400 \mathrm{MHz}$ ): $\delta_{\mathrm{ppm}}=7.45-7.89$ (br), 7.18-7.33 (br), 7.06-7.12 (br), 3.58-3.69 (br), 3.40-3.53 (br), 2.95-3.40 (br), 2.11-2.21 (br), 1.95-2.09 (br), 1.45-1.75 (br), 1.171.21 (br), 0.75-0.93 (br).

\section{Results and discussion}

The chemical structure of $\mathrm{CoA}$ is shown in Figure 1. Because adenosine triphosphate (ATP), ADP and AMP have similar chemical structures as that of CoA, they are the main interferencing species for CoA detection. Except for the similar adenosine and phosphate moieties as those in ATP, ADP and AMP, the CoA molecule also includes a long side chain that is favorable for hydrophobic interactions. At neutral $\mathrm{pH}$, the phosphate groups of the CoA, ATP, ADP and AMP were deprotonated [10], thus the structure of CoA leads to form a complex with oppositely charged conjugated polymer by cooperative electrostatic and hydrophobic interactions, whereas ATP, ADP and AMP form the complexes with oppositely charged conjugated polymer mainly by electrostatic interactions [25]. Previous studies showed that the cationic conjugated polymers could form tight aggregates in the presence of anionic analytes, where the interchain and intrachain interactions of the conjugated polymer lead to fluorescene quenching [34-37]. Thus, by changing the ion strength of assay solution to screen the electrostatic interactions and remain hydrophobic ones, it is

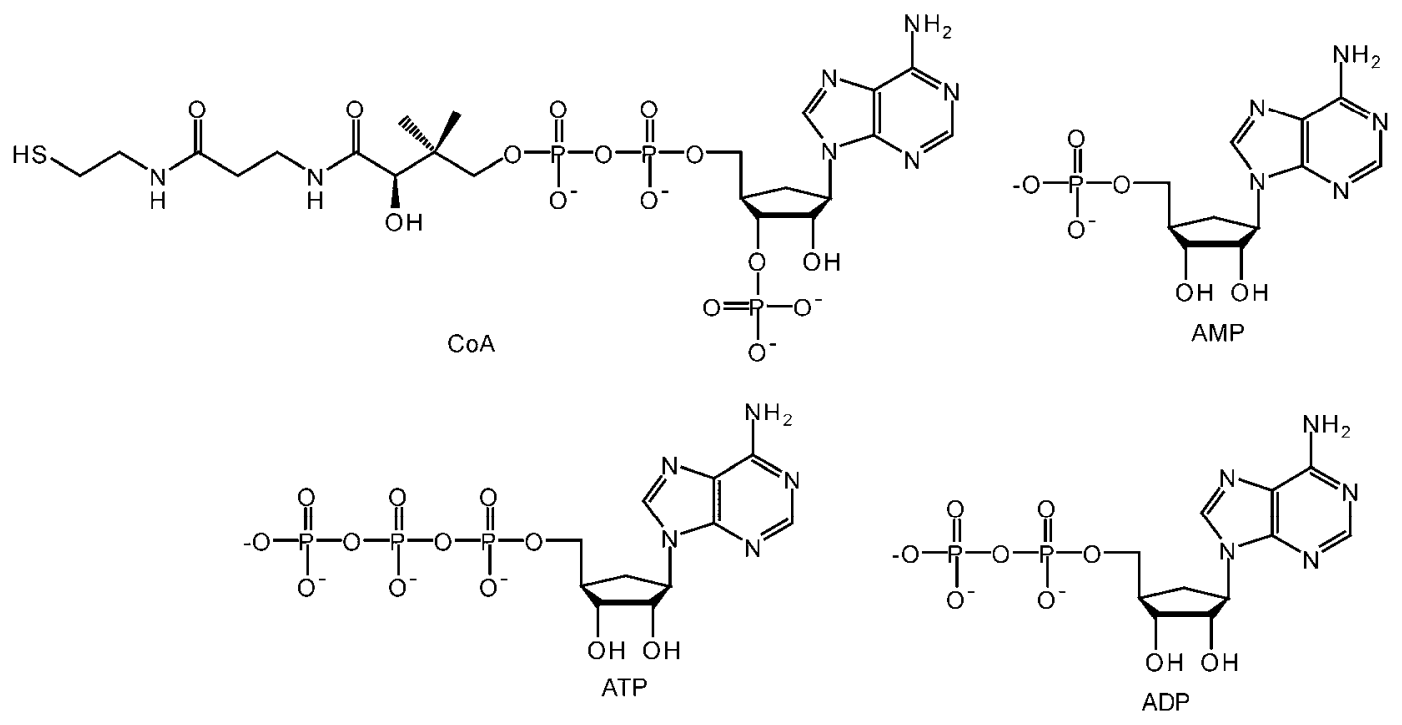

Figure 1 The chemical structures of CoA, AMP, ATP and AMP. 
possible to use cationic conjugated polymer to discriminate CoA from ATP, ADP and AMP by fluorescence measurement.

Figure 2 shows the synthetic entry into the water-soluble cationic conjugated polymer (PFP-PTF). The precursor copolymer 4 were prepared by Suzuki's coupling reaction between one equivalent of monomers $\mathbf{1}$ and $\mathbf{2}$ with $\mathbf{3}$ in the presence of $2.0 \mathrm{~mol} / \mathrm{L} \mathrm{Na}_{2} \mathrm{CO}_{3}$ in water and $\mathrm{Pd}(\mathrm{dppf}) \mathrm{Cl}_{2}$ in THF. GPC analyses show number-average molecular weight of 4 is 15550 amu with PDI of 4.52. The polymer 4 was treated with $33 \%$ trimethylamine aqueous solution to obtain water-soluble cationic PFP-PTF. The photophysical properties of PFP-PTF were investigated in water. The UV-vis absorption spectra of PFP-PTF exhibit maximum peak at $361 \mathrm{~nm}$, which attributes to the $\pi-\pi^{*}$ transition of the polymer backbone. When we excited the solution at $361 \mathrm{~nm}$, the emission spectra show maximum peak at $475 \mathrm{~nm}$.

Figure 3(a) shows the emission spectra of PFP-PTF $([\mathrm{PFP}-\mathrm{PTF}]=4 \mu \mathrm{mol} / \mathrm{L}$ in repeat unit $(\mathrm{RU}))$ with the addition of $\mathrm{CoA}([\mathrm{CoA}]=0-6.5 \mu \mathrm{mol} / \mathrm{L})$ in water with an excitation wavelength of $361 \mathrm{~nm}$. The addition of CoA induces to a obvious quenching of the PFP-PTF emission, and a
$87 \%$ decrease at $475 \mathrm{~nm}$ is obtained. To study the specific interaction of PFP-PTF to CoA, the fluorescence spectra of PFP-PTF in the presence of ATP, ADP and AMP were also investigated under the same condition as CoA. As shown in Figure 3(b), for AMP, a 10\% decrease in PFP-PTF intensity at $475 \mathrm{~nm}$ is observed, and less than $25 \%$ decrease is observed for $\mathrm{ADP}([\mathrm{ADP}]=[\mathrm{AMP}]=6.5 \mu \mathrm{mol} / \mathrm{L})$. This showes that ADP and AMP containing less negative charges in comparison to $\mathrm{CoA}$ do not induce the aggregation of PFP-PTF by electrostatic interactions. For ATP, an obvious quenching of the PFP-PTF emission at $475 \mathrm{~nm}$ is observed, however, the fluorescence quenching efficiency is less than that of CoA in the concentration below $4.0 \mu \mathrm{mol} / \mathrm{L}$. These results show that the PFP-PTF can discriminate CoA from interferencing ATP ADP AMP species.

Above fluorescence results showed intense interpolymer $\pi$-stacking aggregation of PFP-PTF forms in the presence of CoA compared to ATP. Because CoA and ATP have same number of negative charges (four) in neutral water solution, the more efficient fluorescence quenching of PFP-PTF in the presence of $\mathrm{CoA}$ should result from the hydrophobic interactions of the side chains of CoA with PFP-PTF except<smiles>CC1(C)OB(c2ccc(B3OC4(C)C5(C)OC(C)(C5(C)C)C4(C)O3)cc2)OC1(C)O</smiles><smiles>BrCCCCCCC1(CCCCCCBr)c2cc(I)ccc2-c2ccc(I)cc21</smiles>

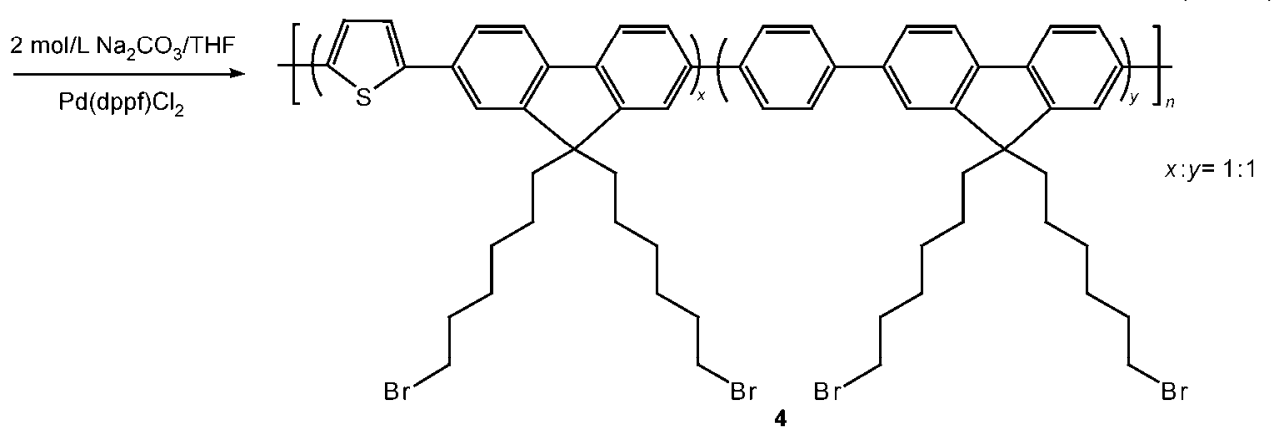
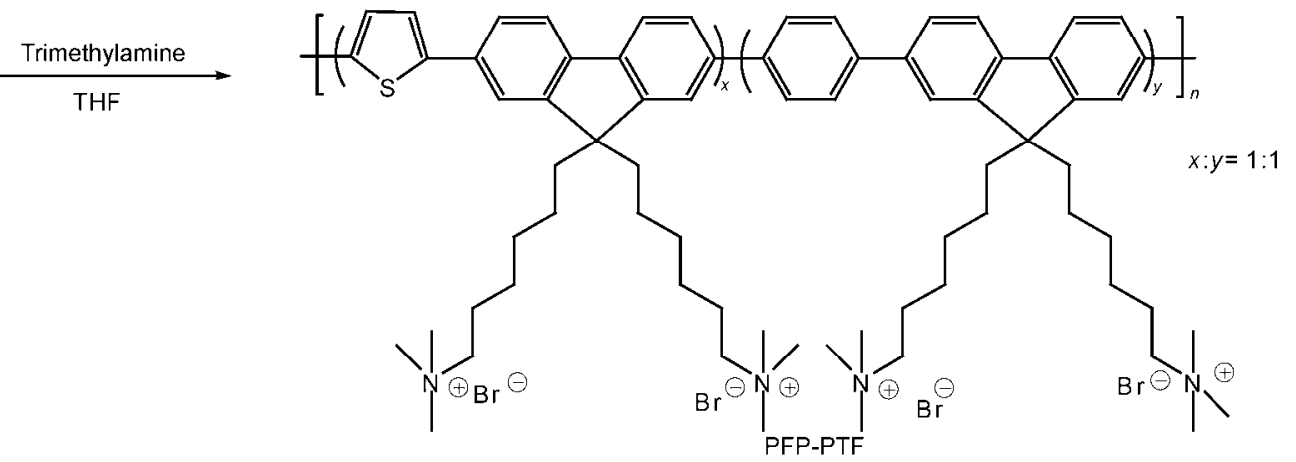

Figure 2 Synthetic routine of polymer PFP-PTF. 

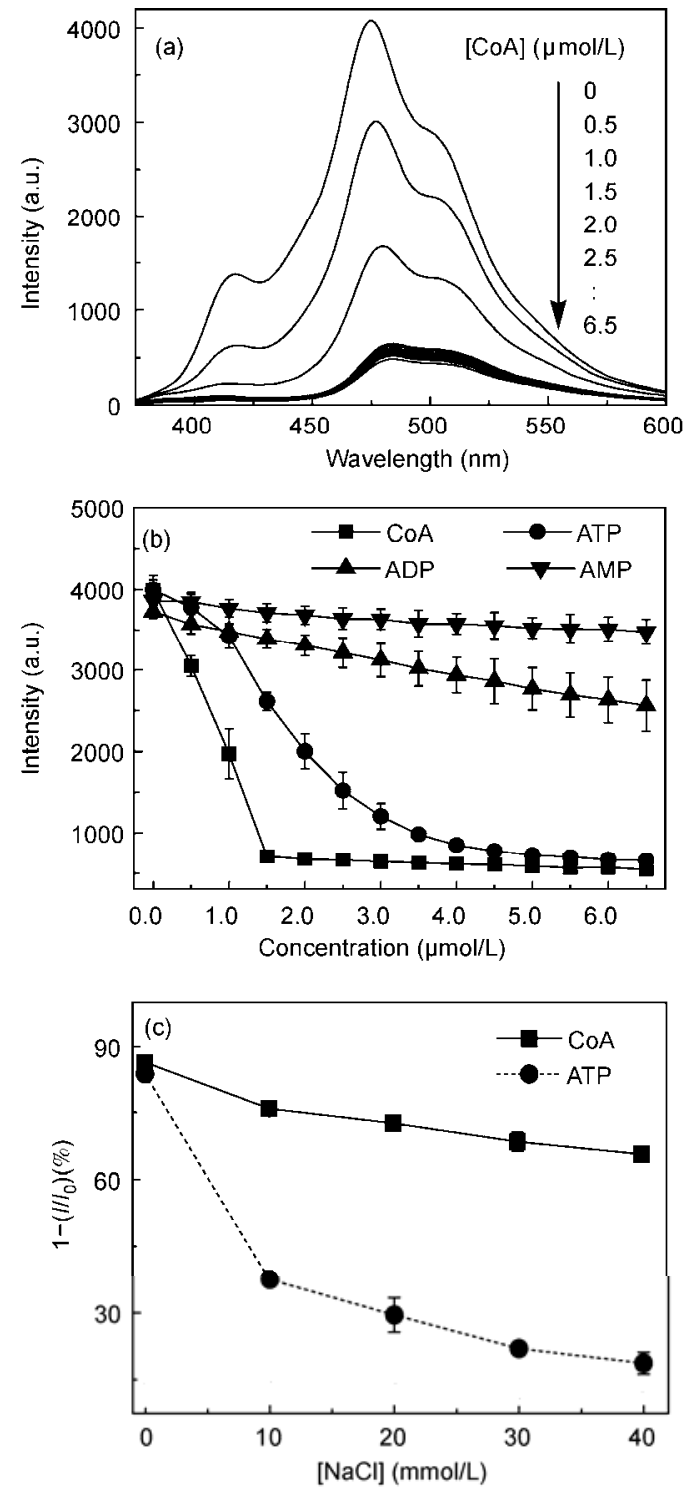

Figure 3 (a) The fluorescence emission spectra of PFP-PTF in $\mathrm{H}_{2} \mathrm{O}$ with CoA. (b) The fluorescence intensity of PFP-PTF at $475 \mathrm{~nm}$ as function of concentrations of CoA, ATP, ADP and AMP. [PFP-PTF] $=4 \mu \mathrm{mol} / \mathrm{L}$, $[\mathrm{CoA}]=[\mathrm{ATP}]=[\mathrm{ADP}]=[\mathrm{AMP}]=0-6.5 \mu \mathrm{mol} / \mathrm{L}$. (c) The fluorescence quenching efficiency of PFP-PTF as function of $\mathrm{NaCl}$. [PFP-PTF] $=$ $4 \mu \mathrm{mol} / \mathrm{L},[\mathrm{CoA}]=[\mathrm{ATP}]=6.5 \mu \mathrm{mol} / \mathrm{L} .[\mathrm{NaCl}]=0-40 \mathrm{mmol} / \mathrm{L}$. The excitation wavelength is $361 \mathrm{~nm}$.

for electrostatic interactions. To give evidence on this quenching mechanism, the dependence of the fluorescence quenching efficiency of PFP-PTF in the presence of CoA and ATP was studied as a function of $\mathrm{NaCl}$ concentration in water solution (Figure 3(c)). With the increase of $\mathrm{NaCl}$ concentration from 0 to $40 \mathrm{mmol} / \mathrm{L}$, the quenching efficiency alters from $87 \%$ to $67 \%$ for CoA, and that for ATP changes from $81 \%$ to $23 \%$. These results furthermore give evidence that the higher ion strength decreases the electrostatic attractions, and the remaining hydrophobic interactions dominate the formation of PFP-PTF/CoA complex.

Noted that in Figure 3(b), in the higher concentrations of
ATP and CoA $(>4.0 \mu \mathrm{mol} / \mathrm{L})$ in water, the similar quenching efficiency of the PFP-PTF emission are observed, where the PFP-PTF can not discriminate CoA from ATP. When the ion concentration of the water solution is inceresed to 40 $\mathrm{mmol} / \mathrm{L}$, the quenching efficiency of PFP-PTF by CoA is three times higher than that by ATP (Figure 3(c)), which makes the PFP-PTF possible to sense CoA from interferencing ATP ADP AMP species even in higher concentrations. Thus, the presence of sodium ions in assay solution improves the selectivity of this method.

The aggregation of PFP-PTF in the presence of CoA under $40 \mathrm{mmol} / \mathrm{L} \mathrm{NaCl}$ is also supported by linear light scattering spectra [38,39], where the electrostatic interactions between them are screened. Figure 4 shows the linear light scattering spectra of PFP-PTF in the presence of CoA, ATP, ADP and AMP in the wavelength range of 200-700 nm. There is no obvious light scattering for PFP-PTF itself. Upon adding CoA, the light scattering intensity of PFP-PTF improves approximately three times in comparison to that of itself, which suggests that the tight aggregation forms in solution in the presence of CoA mainly through hydrophobic interactions. When ATP, ADP and AMP were respectively added, there were not obvious changes of PFP-PTF light scattering intensity because they cannot form compact aggregate with PFP-PTF via electrostatic interactions in this condition. These results are consistent with those of fluorescence measurements. The linear light scattering technique also offers a signal "turn-on" detection of CoA from ATP, ADP and AMP.

\section{Conclusions}

As compared to the earlier published method to determine the CoA by HPLC [12-14], our detection limit is only $0.5 \times 10^{-6} \mathrm{~mol} / \mathrm{L}$, which is much higher than the lowest detection limit of HPLC method was 3 pmol [13], but

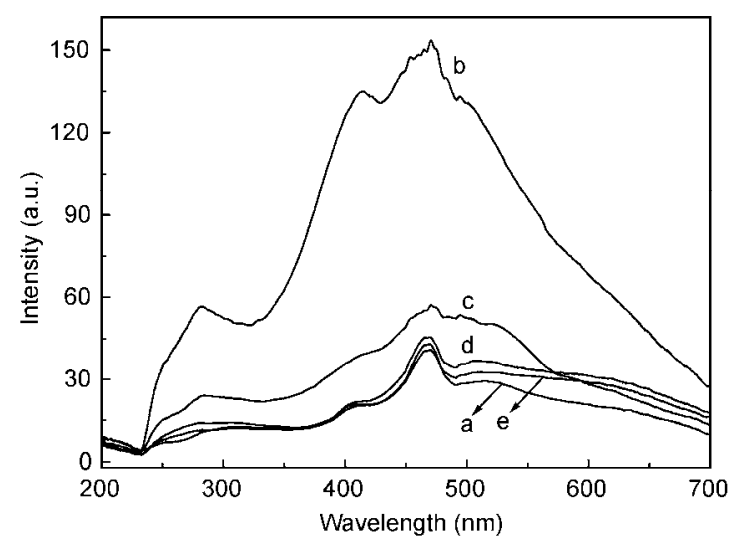

Figure 4 Linear light scattering spectra of PFP-PTF itself (a) and in the presence of (b) CoA, (c) ATP, (d) ADP and (e) AMP in water solution containing $40 \mathrm{mmol} / \mathrm{L} \mathrm{NaCl}$. $[\mathrm{PFP}-\mathrm{PTF}]=4 \mu \mathrm{mol} / \mathrm{L},[\mathrm{CoA}]=[\mathrm{ATP}]=$ $[\mathrm{ADP}]=[\mathrm{AMP}]=6.5 \mu \mathrm{mol} / \mathrm{L}$. The excitation wavelength is $361 \mathrm{~nm}$. 
fluorescence method is more simple and rapid. It makes it possible to directly and real-time detect the concentration of $\mathrm{CoA}$ in the tissues sample without extraction and purification.

In summary, a new cationic conjugated polymer was designed and synthesized to optically discriminate CoA among structurally similar biomolecules, ATP, ADP and AMP. The analyte-induced aggregation of the conjugated polymer by $\pi$-stacking between their main chains leads to the fluorescence quenching. Except for the similar adenosine and phosphate moieties as those in ATP, ADP and AMP, the CoA molecule also includes a long side chain, which leads to form a complex with oppositely charged conjugated polymer by cooperative electrostatic and hydrophobic interactions. The increase of the ion strength of the assay solution screens the electrostatic attractions, and the remaining hydrophobic interactions dominate the formation of PFP-PTF/CoA complex. At this case, the quenching efficiency of PFP-PTF by $\mathrm{CoA}$ is much higher than that by ATP, ADP and AMP, which impart the PFPPTF to sense CoA from these interference species.

This work was supported by the Innovative Research Team of Shaanxi University of Science \& Technology (TD12-04), the Doctoral Scientific Research Fund of Shaanxi University of Science \& Technology (BJ09-15), National Natural Science Foundation of China (51103082).

1 Lipmann F. Les Prix Nobel. Stockholm: 1954. 151

2 Moffatt J G, Khorana H G. The total synthesis of coenzyme A. J Am Chem Soc, 1959, 81: 1265

3 McCormick D B, Wright L D. Methods in Enzymology. 18th ed. London: Academic Press, 1970. 125-133

4 Lee C H, Chen A F, Everse J. The Pyridine Nucleotide Coenzymes. 2nd ed. New York: Academic Press, 1982. 189

5 Martin D P, Drueckhammer D G. Combined chemical and enzymic synthesis of coenzyme A analogs. J Am Chem Soc, 1992, 114: 72877288

6 Shimizu S, Kubo K, Morioka H, et al. Studies on metabolism of pantothenic acid in microorganisms. 9. Some aspects of enzyme activities involved in coenzyme A biosyntheis in various microorganisms. Agr Biol Chem, 1974, 38: 1015-1021

7 Abiko Y. Pantothenic acid and coenzyme A: Dephospho-CoA pyrophosphorylase and dephospho-CoA kinase as a possible bifunctional enzyme complex (ATP: Pantetheine-4'-phosphate adenyltransferase, EC 2.7.7.3 and ATP: Dephospho-CoA 3'-phosphotransferase, EC 2.7.1.2.24). Method Enzymol, 1970, 18A: 358-363

8 Worrall D M, Tubbs P K. A bifunctional enzyme complex in coenzyme A biosynthesis: Purification of pantetheine phosphate adenylyltransferase and dephospho-CoA kinase. Bio Chem J, 1983, 215 : 153-157

9 Tahiliani A G, Beinlich C J. Pantothenic acid in health and disease. Vitam Horm, 1991, 46: 165-228

10 Keire D A, Robert J M D, Rabenstein L. Microscopic protonation equilibria and solution conformations of coenzyme A and coenzyme A disulfides. J Org Chem, 1992, 57: 4427-4431

11 Osono Y, Hirose N, Nakajima K, et al. The effects of pantethine on fatty liver and fat distribution. J Aather Throm, 2000, 7: 55-58

12 Susumu Y, Masaharu N, Hiroyuki W, et al. Specific detection of acetyl-coenzyme A by reversed-phase ion-pair high-performance liquid chromatography with an immobilized enzyme reactor. J Chrom A, 1992, 590: 241-245
13 Abraham D, Alexis G, Daniel K, et al. Rapid method for the separation and detection of tissue short-chain coenzyme A esters by reversed-phase high-performance liquid chromatography. J Chrom B: Biom Sci Appl, 1995, 667: 148-152

14 Martin J, Mangino, John Z, et al. Improved and simplified tissue extraction method for quantitating long-chain acyl-coenzymeA thioesters with picomolar detection using high-performance liquid chromatography. J Chrom B: Bioml Sci Appl, 1992, 577: 157-162

15 Thomas S W, Joly G D, Swager T M. Chemical sensors based on amplifying fluorescent conjugated polymers. Chem Rev, 2007, 107: 1339-1386

16 Liu B, Bazan G C. Homogeneous fluorescence-based DNA detection with water-soluble conjugated polymers. Chem Mater, 2004, 16: 4467-4476

17 Zhu C, Liu L B, Qiong Y, et al. Water-soluble conjugated polymers for imaging, diagnosis, and therapy. Chem Rev, 2012, 112: 4687-4735

18 Feng X L, Liu L B, Wang S, et al. Water-soluble fluorescent conjugated polymers and their interactions with biomacromolecules for sensitive biosensors. Chem Soc Rev, 2010, 9: 2411-2419

19 Duan X R, Liu L B, Feng F D, et al. Cationic conjugated polymers for optical detection of DNA methylation, lesions, and single nucleotide polymorphisms. Acc Chem Res, 2010, 43: 260-270

20 He F, Tang Y, Wang S, et al. Fluorescent amplifying recognition for DNA G-quadruplex folding with a cationic conjugated polymer: A platform for homogeneous potassium detection. J Am Chem Soc, 2005, 127: 12343-12346

21 Feng F, Tang Y, Wang S, et al. Continuous fluorometric assays for acetylcholinesterase activity and inhibition using conjugated polyelectrolytes. Angew Chem Int Ed, 2007, 46: 7882-7886

22 Feng F, He F, An L, et al. Fluorescent conjugated polyelectrolytes for biomacromolecule detections. Adv Mater, 2008, 20: 2959-2964

23 Wosnick J H, Mello C M, Swager T M. Synthesis and application of poly(phenylene ethynylene)s for bioconjugation: A conjugated polymer-based fluorogenic probe for proteases. J Am Chem Soc, 2005, 127: 3400-3405

24 Nilsson K P R, Inganäs O. Chip and solution detection of DNA hybridization using a luminescent zwitterionic polythiophene derivative. Nat Mater, 2003, 2: 419-424

25 An L, Tang Y, Feng F, et al. Water-soluble conjugated polymers for continuous and sensitive fluorescence assays for phosphatase and peptidase. J Mater Chem, 2007, 17: 4147-4152

26 Fan C, Plaxco K W, Heeger A J. High-efficiency fluorescence quenching of conjugated polymers by proteins. J Am Chem Soc, 2002, 124: 5642-5643

27 Pinto M R, Schanze K S. Amplified fluorescence sensing of protease activity with conjugated polyelectrolytes. Proc Natl Acad Sci USA, 2004, 101: 7505-7510

28 Gaylord B S, Heeger A J, Bazan G C. DNA detection using watersoluble conjugated polymers and peptide nucleic acid probes. Proc Natl Acad Sci USA, 2002, 99: 10954-10957

29 Wang S, Gaylord B S, Bazan G C. Fluorescein provides a resonance gate for FRET from conjugated polymers to DNA intercalated dyes. J Am Chem Soc, 2004, 126: 5446-5451

30 Ho H A, Najari A, Leclerc M. Optical detection of DNA and proteins with cationic polythiophenes. Acc Chem Res, 2008, 41: 168-178

31 Bunz U H F. Poly(aryleneethynylene)s: Syntheses, properties, structures, and applications. Chem Rev, 2000, 100: 1605-1644

32 Achyuthan K E, Bergstedt T S, Chen L, et al. Fluorescence superquenching of conjugated polyelectrolytes: Applications for biosensing and drug discovery. J Mater Chem, 2005, 15: 2648-2656

33 Li C, Numata M, Takeuchi M, et al. A sensitive colorimetric and fluorescent probe based on a polythiophene derivative for the detection of ATP. Angew Chem Int Ed, 2005, 44: 6371-6374

34 Sun H, Feng F, Yu M, et al. Analyte-induced aggregation of a water-soluble conjugated polymer for fluorescent assay of oxalic acid. Macromol Rapid Comm, 2007, 28: 1905-1911

35 Kim J, McQuade D T, McHugh S K, et al. Ion-specific aggregation in 
conjugated polymers: Highly sensitive and selective fluorescent ion chemosensors. Angew Chem Int Ed, 2000, 39: 3868-3872

36 Nelson T L, O'Sullivan C, Greene N T, et al. Cross-reactive conjugated polymers: Analyte-specific aggregative response for structurally similar diamines. J Am Chem Soc, 2006, 128: 5640-5641

37 Tang Y, He F, Yu M, et al. A reversible and highly selective fluorescent sensor for mercury (II) using poly(thiophene)s that contain thy- mine moieties. Macromol Rapid Commun, 2006, 27: 389-392

38 Du B, Li Z, Liu C. One-step homogeneous detection of DNA hybridization with gold nanoparticle probes by using a linear light-scattering technique. Angew Chem Int Ed, 2006, 45: 8022-8025

39 Togashi D M, Costa S M B, Sobral A J F N, et al. Self-aggregation of lipophilic porphyrins in reverse micelles of aerosol OT. J Phys Chem B, 2004, 108: 11344-11356

Open Access This article is distributed under the terms of the Creative Commons Attribution License which permits any use, distribution, and reproduction in any medium, provided the original author(s) and source are credited. 